Revista Brasileira de

Engenharia Agrícola e Ambiental

v.14, n.7, p.762-767, 2010

Campina Grande, PB, UAEA/UFCG - http://www.agriambi.com.br

Protocolo 221.08 - 27/11/2008 • Aprovado em 18/02/2010

\title{
Estudo da precipitação mensal durante a estação chuvosa em Diamantina, Minas Gerais
}

\author{
João P. G. Vieira ${ }^{1}$, Maria J. H. de Souza' ${ }^{2}$, Joseane M. Teixeira ${ }^{3}$ \& Felipe P. de Carvalho ${ }^{4}$
}

\begin{abstract}
RESUMO
0 objetivo deste trabalho foi estudar o comportamento da precipitação pluvial no período da estação chuvosa na região de Diamantina, M G. Dados de precipitação pluviométrica mensal, da série histórica de 1977 a 2006, foram utilizados e averiguada a probabilidade para várias classes de precipitação, através da função de frequência acumulada, usando-se a metodologia da distribuição W eibull com averiguação de aderência pelo teste Kolmogorov-Smirnov com nível de significância de 5\%; de classes definidas em função de percentis predeterminados. Os resultados mostram que a estação chuvosa, compreendida entre outubro e março, representa $88 \%$ do total precipitado anual. Os meses de janeiro e dezembro apontam as maiores probabilidades de ocorrência de precipitação com 220,1 mm e $167,8 \mathrm{~mm}$, respectivamente, a nível de $25 \%$ de probabilidade de ocorrência. 0 modelo de distribuição Weibull apresentou bom ajuste da série climatológica para estudos probabilísticos mostrando os parâmetros dentro dos limites estatísticos preestabelecidos.
\end{abstract}

Palavras-chave: agroclimatologia, distribuição WEIBULL, probabilidade de chuva

\section{Study of monthly precipitation during the rainy season in Diamantina, Minas Gerais}

\begin{abstract}
This paper had as its objective the study of the rainfall behavior in the rainy season in the area of Diamantina, MG, Brazil. Monthly rainfall data of historical series from 1977 to 2006 were used. The probability was checked of several rainfall classes through the accumulated frequency function using the W eilbull's distribution methodology with adherence verification by Kolmogorov-Smirnov test with significance level of $5 \%$, classes defined by predetermined percentage. The results show that the rainy season, between 0 ctober and March, represents $88 \%$ of the annual rainfall. The months of January and December presented the largest probabilities of rainfall with $220.1 \mathrm{~mm}$ and $167.8 \mathrm{~mm}$, respectively, at a level of $25 \%$ of occurrence probability. The Weilbull distribution model presented a good adjustment of the climatic series for probabilities studies, showing the parameters within the statistical limits established before.
\end{abstract}

Key words: agriclimatology, Weilbull distribution, rain probability

\footnotetext{
${ }^{1}$ Bolsista PIBIC-FAPEMIG, graduando em Engenharia Florestal/Universidade Federal dos Vales do Jequitinhonha e Mucuri, Rua Cerâmica n.70, Ap. 301, CEP 35930-000, João Monlevade, MG. Fone: (38) 8407 3761. E-mail: joaopaulofloresta@yahoo.com.br

${ }^{2}$ Departamento de Agronomia/UFVJM, Campus II, Rodovia MGT 367 , Km 58, 3 n.5000, Bairro Alto da Jacuba, CEP 39100-000 Diamantina, MG. Fone: (38) 3532 1211. E-mail: mariahatem@yahoo.com.br

3 Departamento de Ciências Básicas/UFVJM, Fone: (38) 3532-1222. E-mail: josianemt@uol.com.br

${ }^{4}$ Graduando em Agronomia/UFVJM, Rua José Aquilar de Paula 45B, Santo Inácio, Diamantina, MG. Fone: (38) 9945 3344. E-mail:felipepaolinelli_dm@hotmail.com
} 


\section{INTRODUÇÃO}

O município de Diamantina, MG, está localizado na região do Espinhaço Meridional, com predominância de solos rochosos e arenosos e com baixo poder de retenção de umidade (água) em termos gerais, segundo Silva et al. (2005).

A precipitação pluvial é responsável por grande parte do desenvolvimento agrário de uma região. Em regiões de recursos hídricos escassos, a contribuição de produtos para a rede alimentícia é mínima devido a problemas meteorológicos; por outro lado, a precipitação pluvial é a forma mais econômica e ambientalmente correta de uso da água na agricultura, pois não há necessidade de se importar água através de irrigação, além de manter em equilíbrio os recursos hídricos existentes em outras regiões diminuindo, consequentemente os custos de produção, mas preservando o meio ambiente.

Na maior parte das terras agricultáveis não se utiliza a irrigação, dependendo-se exclusivamente dos regimes pluviométricos. Segundo Castro (1994) utiliza-se no Brasil, a precipitação mensal média no dimensionamento de projetos agrícolas, fato este passível de ocasionar um erro para o produtor devido à grande variabilidade dos dados. De conformidade com Mantovani et al. (2006) e Bernardo (1995), a precipitação mensal máxima utilizada a de $75 \%$ de probabilidade resultando, portanto, uma precipitação mínima de $25 \%$, haja vista tratar-se de um valor inverso do outro, em termos probabilísti$\cos$.

Araújo et al. (2001) e Mello et al. (2003), afirma que vários modelos vêm sendo usados no meio meteorológico para estimativas de distribuição pluviométricas, visando à estimativa de precipitação provável para diferentes locais e períodos de ocorrência. A precipitação pluvial de determinado local pode ser estimada, dentre outras formas, em termos probabilísticos, mediante modelos teóricos de distribuição ajustados a uma série de dados. Segundo Silva et al. (2007), dentre os modelos de distribuição o modelo de distribuição Weibull demonstra resultados satisfatórios, não apresentando diferença significativa quando comparado com outros métodos de distribuição, como função gama e log-normal, para períodos da estação chuvosa.

De acordo com Vieira (2007), a precipitação de Diamantina se concentra no semestre de outubro a março, sendo que $86 \%$ da precipitação anual se encontram nesse período. Ficou evidenciada, no presente estudo, a distribuição da precipitação ao longo da estação chuvosa, porém sem um estudo estatístico com subsídio probabilístico.

Devido à falta de informações probabilísticas que envolvem as características do regime de chuva na região de Diamantina, torna-se necessário um estudo mais profundo que evidencie o comportamento da precipitação dentro da estação chuvosa, a fim de subsidiar as atividades agrícolas e orientar os programas de pesquisas agrometeorológicas afins da região, além de identificar a intensidade de chuva na região de acordo com Silva et al. (2007). Objetivou se com o presente trabalho ajustar uma distribuição de probabilidades mensais e realizar estimativas probabilísticas de precipitação mensal do período de outubro a março para a região de Diamantina, MG.

\section{MATERIAL E MÉTODOS}

O trabalho foi desenvolvido nas dependências da Universidade Federal dos Vales do Jequitinhonha e Mucuri (UFVJM), utilizando-se dados diários de precipitação obtidos junto ao $5^{\circ}$ Distrito de Meteorologia $-5^{\circ}$ DISME, pertencente ao Instituto Nacional de Meteorologia - INME, além dos dados publicados nas normais climatológicas referentes a esta mesma estação, Brasil (2006).

A cidade de Diamantina se encontra a $1296 \mathrm{~m}$ de altitude, com latitude de $18,15^{\circ} \mathrm{S}$ e longitude de $43,36^{\circ} \mathrm{W}$; seu clima é Cwb, conforme a classificação Köppen, ou seja, temperado úmido, com inverno seco e chuvas no verão, cuja temperatura do mês mais quente é inferior a $22^{\circ} \mathrm{C}$, de acordo com Souza (2006). Considerada pela Uited Nations Educational, Scientific and Cultural Organization, patrimônio histórico mundial desde 1999, UNESCO (2008), Diamantina apresenta grande movimentação turística, razão por que a precipitação é de suma importância para o reabastecimento de seus mananciais de água que, além da população local, tem de atender aos turistas que a frequentam.

A estação climatológica do INMET está localizada na latitude de $18,25^{\circ} \mathrm{S}$, longitude de $43,60^{\circ} \mathrm{W}$ e altitude de 1296,9 m; também se utilizaram dados de precipitação diária fornecidos pela Agência Nacional das Águas (ANA), localizada na latitude de $17,61^{\circ} \mathrm{S}$, longitude de $43,60^{\circ} \mathrm{W}$ e altitude de $1300 \mathrm{~m}$.

$\mathrm{O}$ trabalho se baseou em dados mensais obtidos com o somatório dos dados diários, de outubro a março, de precipitação pluviométrica de um período de 30 anos (1977 a 2006). Empregaram-se os dados da ANA com o intuito de preencher as lacunas referentes à série histórica do INMET, em detrimento dos meses de fevereiro e março do ano 1979 a julho de 1990. Os dados utilizados se referiam à precipitação diária ocorrida das $9 \mathrm{~h}$ do dia anterior às $9 \mathrm{~h}$ do dia seguinte, conforme padronização internacional da Organização Meteorológica Mundial (OMM), ou seja, o volume de água precipitado ocorrido entre as $9 \mathrm{~h}$ do dia anterior (n-1) e $9 \mathrm{~h}$ do dia seguinte.

Para os anos com dados faltantes, levantaram-se estudos baseados na série temporal, o que possibilitou a estimativa desses dados objetivando-se obter, assim uma série climatológica completa, auxiliando ainda mais no processamento dos dados.

Para o cálculo de função de distribuição (fd) de probabilidade Weibull utilizou-se a metodologia apresentada por Catalunha et al. (2002). Determinaram-se os parâmetros para cada sequência de mês considerando-se os valores de precipitação mensal e se averiguou, para o teste da fd, a normalidade dos dados pelo teste Shapiro-Wilk (SH), segundo Assis et al. (1996). Para a sequência de meses em que a distribuição não apresentou normalidade, fez-se uso do teste para dados não paramétricos Kolmogorov-Smirnov (KS), de acordo com D'Agostino \& Stephens (1986), Assis et al. (1996). O teste de Kolmogorov-Smirnov pode ser usado tanto para dados agrupados quanto para dados individuais. De acordo com Catalunha et al. (2002), nos dados agrupados não há restrição quanto ao número nem ao valor das classes, que é baseado no 
módulo da maior diferença entre a probabilidade observada e a estimada que, por sua vez, é comparada com um valor tabelado, de acordo com o número de observações da série testada, evento que evita o aspecto cumulativo de erros.

Para se fazer o ajuste do modelo de fd Weibull por aproximação linear, determinaram-se as classes de precipitação baseadas na distribuição da precipitação de cada seqüência de mês estudada; a partir daí se definiu, para determinar as classes, o uso de percentis, metodologia esta mostrada em Xavier (2001), sendo que os percentiis usados foram 0,$15 ; 0,35$; 0,$50 ; 0,75$ e 0,99 . O emprego dos percentiis preestabelecidos facilita o ajuste e a análise dos dados além, de início, o mesmo número de meses para cada classe estipulada, assim na hora do ajustamento da fd os valores de cada classe de percentil vão ser ajustados da melhor forma devido, principalmente, ao fato de se levar em consideração, no ajustamento dos dados, os valores das classes de precipitação linearizados, a fim de facilitar os testes de normalização.

Para melhor ajuste dos dados e determinação dos parâmetros pela regressão, os dados mensais foram transformados em raiz quadrada e raiz cúbica. Para o teste de normalidade e dados não- paramétricos usaram-se o software $R$ versão 2.7.0 e o software Assistat 7.5 beta.

A função de distribuição Weibull tem como objetivo, ajustar os dados obtidos junto à estação meteorológica para estimar as probabilidades de ocorrência de determinadas classes de precipitação. Esta função de distribuição leva em consideração os valores superiores da classe de precipitação, isto é:

$$
\mathrm{F}(\mathrm{X})=1-\mathrm{e}^{\wedge}\left((\mathrm{x} / \beta)^{\wedge} \gamma\right)
$$

em que $\mathrm{F}(\mathrm{X})$ é a probabilidade de ocorrência de um valor de precipitação mensal menor ou igual a X; para estipular o $\mathrm{X}$, o primeiro passo consiste em se obter o $\mathrm{Z}$, que é calculado através da fórmula:

$$
\mathrm{Z}=\mathrm{Ln}(\mathrm{X})
$$

em que $\mathrm{X}$ é o valor da classe de precipitação. Após identificação do $\mathrm{Z}$ que é o algarismo neperiano da probabilidade de ocorrência de um valor de precipitação de cada classe préestipulada pelo uso dos percentiis, determina-se a variável $\mathrm{Y}$ que pode ser descrita como:

$$
\mathrm{Y}=\left\{\operatorname{Ln}\left\{-\operatorname{Ln}\left[1-\mathrm{F}\left(\mathrm{X}_{\mathrm{i}}\right)\right\}\right]\right.
$$

em que $X_{1}$ é a frequência acumulada observada da série de dados de precipitação mensal, estipulada pelo uso de percentiis.

Após se encontrar as variáveis $\mathrm{X}$ e Y, usou-se o método de mínimos quadrados ordinários para se chegar aos coeficientes de cada classe de precipitação mensal, isto posto, fezse a regressão linear para se chegar aos parâmetros â e á.

\section{RESULTADOS E DISCUSSÃO}

Apresentam-se, na Figura 1, os valores acumulados mensais de precipitações médias do período de 1977 a 2006. De

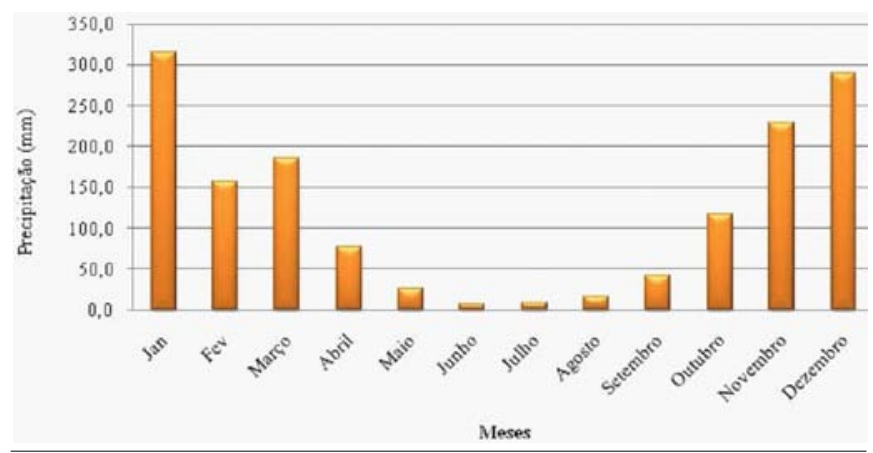

Figura 1. Distribuição anual da precipitação em Diamantina, MG

acordo com a análise da série climatológica, a estação chuvosa compreende o período de outubro a março e apresenta uma precipitação média de 1292,6 $\mathrm{mm}^{2}$ ano $^{-1}$ enquanto a precipitação total anual é de $1468 \mathrm{~mm}$.

Evidencia-se, na Figura 1, a importância do estudo probabilístico de precipitação pluviométrica, pois se observa a distribuição da precipitação durante o ano e a sua concentração no período de outubro a março, representando $88 \%$ de toda a precipitação pluviométrica anual, valores que acompanham os citados por Vieira (2007), apresentam diferença de dois pontos percentuais, a qual é aceitável devido à implementação dos dados faltantes pelo estudo das séries temporais; ainda se pode notar, nesta figura, que durante a sexta chuvosa o mês de maior precipitação mensal é janeiro, apresentando altura de chuva de $315 \mathrm{~mm}$; já o mês de outubro apresenta a menor altura, ou seja, $116 \mathrm{~mm}$. Segundo Carvalho et al. (2008), Diamantina mostra uma estação seca e chuvosa bem definida; desta forma, o período chuvoso compreende os meses de outubro a março e o período seco, os meses de abril a setembro.

Tem-se, na Tabela 1, as médias mensais durante a estação chuvosa, de outubro a março, os desvios padrão, os coeficientes de variação $(\mathrm{CV})$, erro padrão e valores extremos de precipitação pluviométrica mensal (mínimo e máximo) e o ano de ocorrência desses eventos extremos, durante o período de 1977 a 2006. Verifica-se, nesta tabela, que o CV das médias mensais foi elevado, evidenciando a variabilidade da precipitação sendo que no mês de dezembro se obteve o menor CV (35\%), enquanto o maior foi em fevereiro (76\%) o que tam-

Tabela 1. Médias mensais, desvio-padrão, coeficiente de variação (CV), erro padrão e valores extremos de precipitação

\begin{tabular}{|c|c|c|c|c|c|c|c|c|}
\hline Mês & $\begin{array}{l}\text { Média } \\
\text { (mm) }\end{array}$ & $\begin{array}{c}\text { Desvio } \\
\text { Padrão } \\
\text { (mm) }\end{array}$ & CV(\%) & $\begin{array}{l}\text { Erro } \\
\text { Padrão } \\
\text { (mm) }\end{array}$ & $\begin{array}{c}\text { Mínimo } \\
(\mathrm{mm})\end{array}$ & $\begin{array}{c}\text { Ano } \\
\text { ocorrido }\end{array}$ & $\begin{array}{c}\text { Maximo } \\
(\mathrm{mm})\end{array}$ & $\begin{array}{c}\text { Ano } \\
\text { ocorrido }\end{array}$ \\
\hline Outubro & 116,3 & 75,4 & 65 & 13,8 & 11 & 1994 & 361,7 & 2006 \\
\hline Novembro & 228,3 & 100,3 & 44 & 18,3 & 80,3 & 1993 & 412,8 & 2006 \\
\hline Dezembro & 289,0 & 102,3 & 35 & 18,6 & 144,2 & 1982 & 571,6 & 1989 \\
\hline J aneiro & 315,2 & 194,9 & 62 & 35,6 & 31,1 & 1990 & 684,0 & 1991 \\
\hline Fevereiro & 157,4 & 118,9 & 76 & 21,7 & 13,4 & 2001 & 547,3 & 1979 \\
\hline Março & 185,9 & 138,6 & 75 & 25,3 & 4,2 & 1986 & 552,6 & 1994 \\
\hline $\begin{array}{l}\text { Estação } \\
\text { Chuvosa }\end{array}$ & 1292,6 & 121,7 & 59 & 22,2 & - & - & - & - \\
\hline
\end{tabular}
pluviométrica e ano de ocorrência em Diamantina, MG, para 0 período de 1977 a 2006 
bém pode ser verificado na Tabela 1; assim, do ponto de vista estatístico, a variabilidade dos dados de precipitação se mostra inadequada para o planejamento agrícola, incentivando estudos mais aprofundados com subsídios estatísticos, a fim de se obter dados mais confiáveis.

A Tabela 2 mostra os parâmetros $\alpha$ e $\beta$ da função de distribuição Weibull calculados pelo método de aproximação linear para precipitação mensal em Diamantina, MG; observando-a, verifica-se, com base nos parâmetros alfa $(\alpha)$ e beta $(\beta)$, estimados pelo método de aproximação linear, a possibilidade do uso da função de distribuição Weibull. Os valores de $\alpha$ e $\beta$ assim ajustados, apresentaram-se dentro do nível de significância aceitável sendo que, de acordo com Araújo et al. (2001), os níveis de significância de $95 \%$ são recomendados para este caso. Os parâmetros mostraram bom ajuste devido apenas principalmente, ao estudo do período da estação chuvosa visto que, por não haver valores nulos que, geralmente, são encontrados em meses da estação seca, a normalização dos dados pela raiz quadrada e cúbica foi satisfatória para o teste de aderência Kolmogorov-Smirnov, situação semelhante foi verificada no trabalho de Catalunha et al. (2002), em que se obtiveram melhores ajustes da função de distribuição Weibull no período da estação chuvosa.

Tabela 2. Parâmetros $\alpha$ e $\beta$ da distribuição W eibull calculados pelo método de aproximação linear para precipitação mensal em Diamantina, MG

\begin{tabular}{ccc}
\hline Meses & $\boldsymbol{\alpha}$ & $\boldsymbol{\beta}$ \\
Outubro & 11,14040 & 2,24279 \\
Novembro & 12,97335 & 2,52328 \\
Dezembro & 21,48355 & 2,60390 \\
Janeiro & 10,23366 & 2,65171 \\
Fevereiro & 8,30594 & 2,34377 \\
Março & 8,09031 & 2,43346 \\
\hline
\end{tabular}

Os níveis de probabilidade e seus respectivos dados de precipitação, se encontram na Tabela 3, segundo a distribuição Weibull. Obteve-se um ajuste satisfatório dos valores mensais de precipitação pluviométrica à distribuição Weibull com teste de aderência pelo método de Kolmogorov-Smirnov para os meses de outubro a março, período que compreende a estação chuva da região de Diamantina (Vieira, 2007). Para projetos agrícolas, Bernardo (1995) e Mantovani et al. (2006) recomendam o nível de $75 \%$ de probabilidade de ocorrência

Tabela 3. Probabilidade de ocorrência de precipitação pluvial $(\mathrm{mm})$ em Diamantina com seis níveis de probabilidade

\begin{tabular}{ccccccc}
\hline \multirow{2}{*}{ Meses } & $\mathbf{7}$ Precipitação provável (\%) \\
\cline { 2 - 7 } & $\mathbf{5}$ & $\mathbf{1 0}$ & $\mathbf{2 5}$ & $\mathbf{5 0}$ & $\mathbf{7 5}$ & $\mathbf{9 5}$ \\
Outubro & 25,9 & 38,0 & 65,2 & 104,7 & 151,9 & 229,8 \\
Novembro & 65,7 & 90,8 & 145,0 & 220,5 & 300,4 & 428,7 \\
Dezembro & 136,0 & 165,8 & 220,1 & 281,6 & 341,5 & 423,5 \\
J aneiro & 64,0 & 92,0 & 167,8 & 281,5 & 421,2 & 666,9 \\
Fevereiro & 19,4 & 32,9 & 67,5 & 127,0 & 210,0 & 366,4 \\
Março & 23,3 & 39,3 & 85,6 & 158,3 & 264,6 & 468,7 \\
\hline
\end{tabular}

de chuva, em que a precipitação obtida nesse nível é mais confiável para o dimensionamento de projetos agrícolas e de irrigação. Verifica-se, observando-se a Tabela 3, que no mês de janeiro existe a perspectiva, a nível de $75 \%$, de se ter uma precipitação mensal acumulada inferior a 421,6 mm e $25 \%$ de probabilidade de ocorrer um valor superior a este; já no mês de outubro se pode dizer que há $25 \%$ de chance de um total mensal precipitado superior a $151,9 \mathrm{~mm}$, ou seja, existem $75 \%$ de probabilidade de ocorrer, nesse mês, um valor mensal acumulado inferior a $151,9 \mathrm{~mm}$.

Pode-se constatar, através da análise dos valores apresentados na Tabela 3, a importância da estimativa de parâmetros usando-se alguma função de distribuição probabilística, pois mesmo sendo janeiro o mês de maior quantidade de precipitação mensal (Figura 1) a um nível inferior de probabilidade, outros meses apresentam valores de precipitação superiores a este mês; esta ocorrência pode ser justificada principalmente pelo estudo separado de cada série climatológica, estimando-se parâmetros com subsídios estatísticos se ajustando a fórmula de distribuição, de acordo com o comportamento de cada série de dados de seus respectivos meses.

A Figura 2 apresenta a distribuição de ocorrência de todas as probabilidades de precipitação pluvial do período chuvoso. Medina \& Leite (1984) sugeriram que se fizessem cálculos e estimativas mais seguras para o planejamento agrícola; o recomendado neste caso é usar o nível de probabilidade de $75 \%$, isto é, o mesmo que dizer que existem $25 \%$ de chances de ocorrer um valor máximo acumulado de precipitação para determinado mês. Para o estudo em questão se estimaram as precipitações máximas a nível de $25 \%$ isto, há $25 \%$ de chances de ocorrer determinada precipitação máxima ou se pode dizer, também, que existem $75 \%$ de chances de que ocorram valores inferiores à precipitação determinada; além disso, uma chuva só pode ser considerada significativa quando acima de $50 \mathrm{~mm}^{\text {mês }}{ }^{-}$ 1 de acordo com Vieira (2007).

Constatou-se que as prováveis chuvas com probabilidade de excedência confiável (até 25\%) para cada mês, ocorreram em: outubro $65,2 \mathrm{~mm}$, novembro $90,8 \mathrm{~mm}$, dezembro $220,1 \mathrm{~mm}$, janeiro $167,8 \mathrm{~mm}$, fevereiro $67,5 \mathrm{~mm}$ e março, $85,6 \mathrm{~mm}$, ou seja, espera-se que, em uma sequência de quatro anos, em três deles ocorra uma precipitação igual ou maior aos valores apresentados; essas informações mensais são de extrema importância para o dimensionamento correto de sistemas de irrigação e, acrescidas dos conhecimentos fisiológicos das plantas, constituem bases fundamentais para um manejo eficiente e satisfatório das culturas, em especial na época de semeadura, para evitar déficit hídrico e perdas na produtividade.

O conhecimento mensal do regime pluviométrico levará o produtor rural a direcionar o início e o término do seu planejamento agrícola para o período em que a chuva em excesso ou déficit não prejudique as atividades de semeadura, plantio e atividades correlacionadas evitando assim, perdas significativas com sementes, mudas e queda de rendimentos operacionais de mão-de-obra e máquinas. 
Outubro

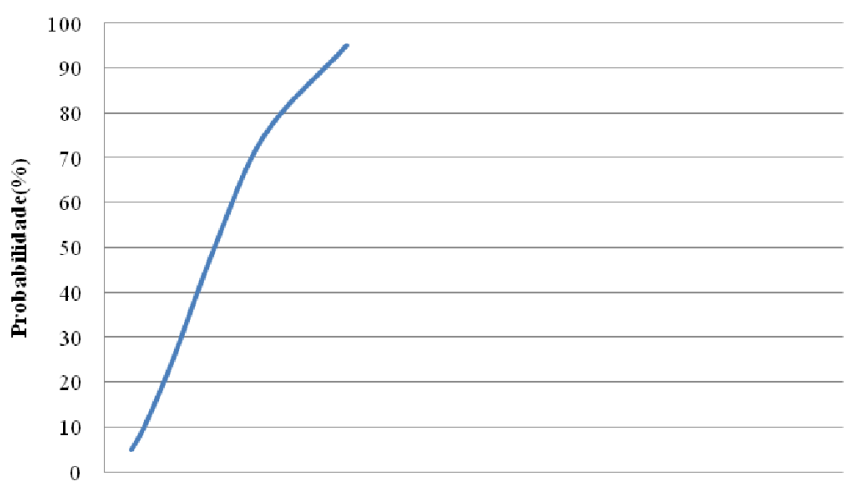

Dezembro

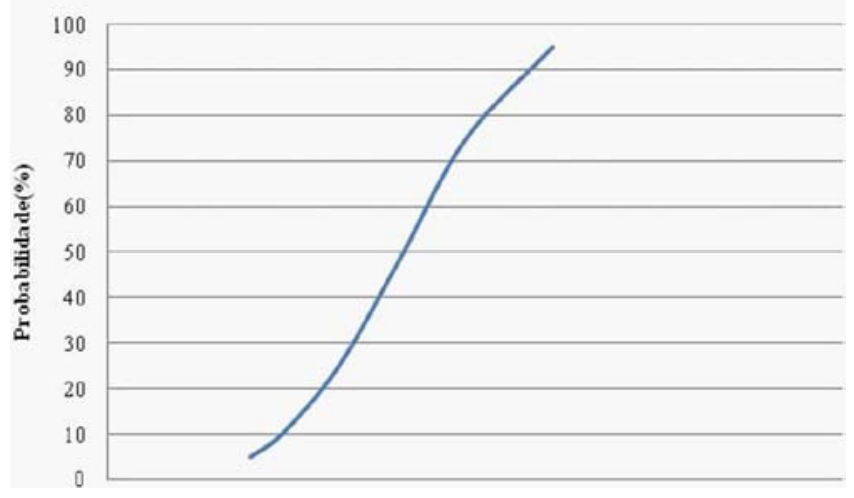

Fevereiro

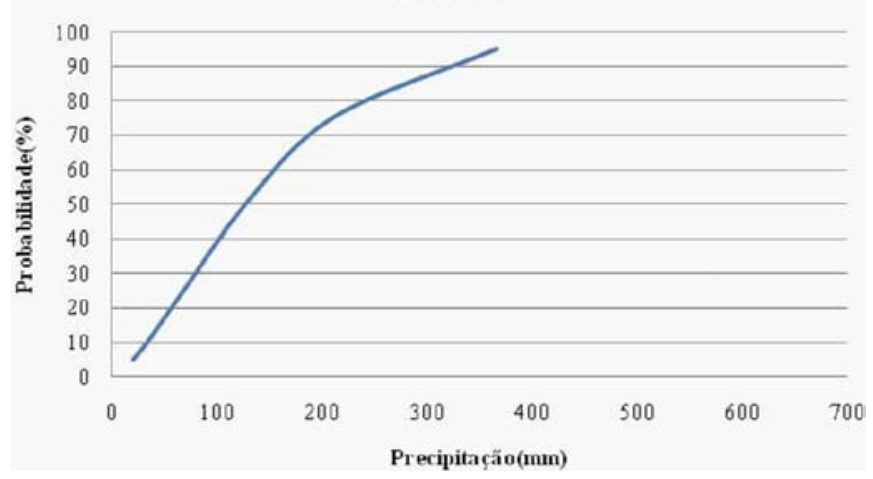

Novembro

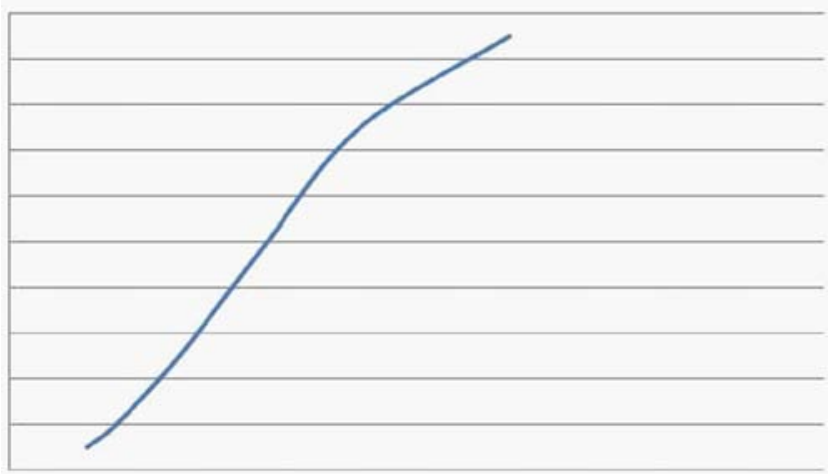

Janeiro

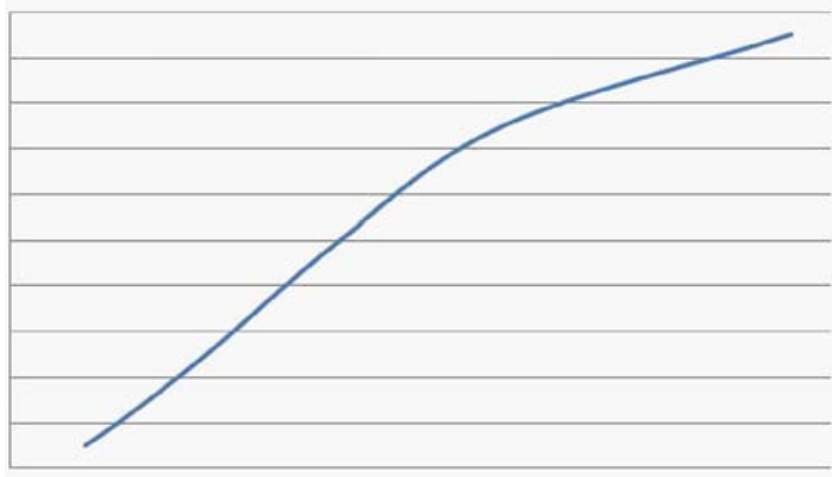

Marco

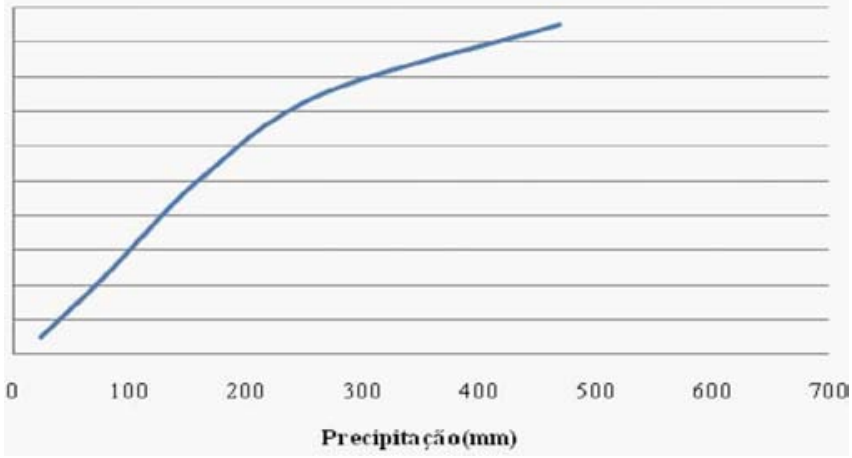

Figura 2. Distribuição da probabilidade de precipitação no município de Diamantina, MG , no período de 1977 a 2006

\section{CONCLUSÕES}

1. O período de outubro a março apresenta níveis de precipitação mínima para atender a maioria das culturas agrícolas, sendo que o montante precipitado desse período equivale a $88 \%$ do total da precipitação anual.

2. Os meses de janeiro e dezembro apresentaram os maiores valores de precipitação mensal a nível de $25 \%$ de probabilidade mínima de ocorrência, gerando valores de 220,1 e 167,8 $\mathrm{mm}$, respectivamente.

3. A função de distribuição Weibull ajustou-se bem aos dados mensais estudados, gerando parâmetros satisfatórios, constituindo-se em uma boa ferramenta para cálculos probabilísticos em precipitação em Diamantina, MG.

\section{LITERATURA CITADA}

Araújo, W. F.; Andrade, A. S. J.; Medeiros, R. D.; Sampaio, R. S. Precipitação pluviométrica mensal provável em Boa Vista, estado de Roraima, Brasil. Revista Brasileira de Engenharia Agrícola e Ambiental, v5, n.3, p.563-567, 2001.

Assis, F. N.; Arruda, H. V. de; P. A. R.. Aplicações de estatística à climatologia: teoria e prática. Pelotas: UFPel, 1996.161p.

Bernardo, S.. Manual de irrigação. 6 ed. Viçosa: Imprensa Universitária, 1995. 657 p. Brasil, Ministério da Agricultura e Reforma Agrária. Secretaria Nacional de Irrigação. Departamento Nacional de Meteorologia. Brasília 2006. 348p. Normais climatológicas (1977-2006). 
Carvalho, F. P.; Souza, M. J. H.; Vieira, J. P. G. Estudo da estiagem em Diamantina, Minas Gerais. In: Jornada Científica e Tecnológica da Universidade Federal dos Vales do Jequitinhonha Mucuri, 2008, Diamantina. Anais... Diamantina: UFVJM, CD Rom.

Castro, R. Distribuição probabilística de freqüência de precipitação na região de Botucatu, SP. Botucatu: UNESP, 1994. 101p. Dissertação Mestrado.

Catalunhaa, M. J.; Sediyama, G. C.; Leal, B. G.; Soares, C. P. B.; Ribeiro, A.. Aplicação de cinco funções densidade de probabilidade a séries de precipitação pluvial no Estado de Minas Gerais. Revista Brasileira de Agrometeorologia, v.10, n.1, p.153$162,2002$.

D'Agostino, R. B., Stephens, M. A. Goodness-of-fit techniques. New York: Marcel Dekker, 1986. 286p.

Mantovani, E. C.; Bernardo, S.; Palaretti, L. F. Irrigação: Princípios e métodos. 6 ed. Viçosa: Imprensa Universitária, 2006. 318p.

Medina, B. F.; Leite, J. A.. Probabilidade de chuva em Boa Vista RR. Pesquisa Agropecuária Brasileira, v.12, p.1437-1441, 1984.

Mello, C. R.; Silvar, A. M.; Lima, J. M.; Ferreira, D. F.; Oliveira,M. $\mathrm{S}$. Modelos matemáticos para predição da chuva de projeto para regiões do Estado de Minas Gerais. Revista Brasileira de Engenharia Agrícola e Ambiental, v.7, n.1, p.121-128, 2003.
Silva, A. C.; Pedreira, L. V. S. F.; Abreu, P. A. A.. Serra do Espinhaço Meridional, paisagens e ambientes. Diamantina: UFVJM, 2005. 271p.

Silva, J. C.; Heldwein, A. B.; Fabrina, B.; Trentin, G.; Edenir, L. Análise de distribuição de chuva para Santa Maria, RS. Revista Brasileira de Engenharia Agrícola e Ambiental, v.11, n.1, p.67-72, 2007.

Souza, M. J. H. Potencialidade climática para a viticultura em Diamantina - MG. In: XI Reunião Argentina de Agrometeorologia, 11, 2006, La Plata, Buenos Aires. Anais.... La Plata, Buenos Aires: Sociedade Argentina de Agrometeorologia, 2006. CD_Rom.

UNESCO - Uited Nations Educational, Scientific and Cultural Organization. Historic centre of the town of Diamantina. http://whc.unesco.org/en/list/890. 12 Nov. 2008.

Vieira, J. P. G. Probabilidades mensais de ocorrência de chuva na região de Diamantina, MG. In: Congresso Brasileiro de Agrometeorologia, Aracaju,Anais,Aracaju:Sociedade Brasileira de Agrometeorologia, 2007, CD-Rom.

Xavier, T. M. B. S.Tempo de chuva: Estudos climáticos e de previsão para o Ceará e Nordeste sentrentrional.Ceará: ABC Editora, 2001.478p. 